\title{
BESPRECHUNGSAUFSATZ
}

\section{Der Streit um die Falkland-Inseln: Welche Lehren? Der argentinisch-britische Konflikt im Spiegel zweier Neuerscheinungen*}

von Hermann Weber

Wenige Kriege dieses Jahrzehnts haben die Welt so in Erstaunen versetzt und das Vertrauen der Öffentlichkeit in den Bestand fundamentaler internationaler Rechtsprinzipien so stark erschüttert wie der zwischen Argentinien und Großbritannien 1982 geführte Krieg um die Rechte an den Falkland-Inseln. Kriege von erheblich längerer Dauer und mit weit höheren Verlusten auf beiden Seiten der Kriegsführenden, wie der Iran/IrakKonflikt oder der Bürgerkrieg in Afghanistan mit massiver Unterstützung durch die Sowjetunion erregten weit geringere Aufmerksamkeit als der Falkland-Krieg. Der Grund für das unterschiedliche Interesse lag in einer Fehlinterpretation heute möglicher Konfliktmuster: ein Krieg innerhalb des westlichen Lagers wegen eines historischen Streites über die Zugehörigkeit einer strategisch uninteressant gewordenen und in ihren wirtschaftlichen Perspektiven eher unattraktiven Inselgruppe im Südatlantik war allgemein für politisch unmöglich und unrealistisch gehalten worden. Es herrschte bis zum Ausbruch des Krieges die Úberzeugung vor, daß die Konfliktmuster von zwei Typen bewaffneter Auseinandersetzungen bestimmt würden: zum einen vom, wenn auch nur theoretisch denkbaren, nuklearen Schlagabtausch der Supermächte, zum anderen von den Bürgerkriegen und regionalen Hegemonialkriegen der Dritten Welt, die wegen der tatsächlichen oder stets möglichen direkten oder indirekten Beteiligung der Großmächte an ihnen den interantionalen Frieden insgesamt labil halten. Der Falkland-Krieg 1982 hat aber gezeigt, daß auch im westlichen Lager eine Bereitschaft bestehen kann, politische Konflikte, denen keine existenzielle oder das vitale Interesse einer Nation berührende Streitfrage zugrundeliegt, auf eine Eskalationsebene zu heben oder dorthin treiben zu lassen, wo man meint eine zusätzliche Option der Konfliktbeendigung, nämlich die Konfliktaustragung mit Waffengewalt, zu haben.

* The Falklands War. Lessons for Strategy, Diplomacy and International Law, ed. by Alberto R. Coll and Anthony C. Arend, Boston 1985, 252 S., Verlag: Allen \& Unwin, $\mathfrak{E} 7.95$; Rudolf Dolzer: Der völkerrechtliche Status der Falkland-Inseln (Malvinas) im Wandel der Zeit, Heidelberg 1986, 239 S., Verlag: R. v. Dekker \& C. F. Müller, DM 98,-. 
Wie wenig Rationalität das militärische Falkland-Abenteuer von 1982 realiter aufwies, wird aus den Zahlenangaben deutlich, die das britische Verteidigungsministerium veröffentlicht hat. Danach haben die gesamten Ausgaben zur Wiederherstellung und Wiederbefestigung des von Argentinien verletzten status quo den britischen Staatshaushalt bis März 1986 mit 2,6 Milliarden Pfund (= 7 Milliarden Mark) belastet. Darin sind die militärischen Verteidigungsausgaben, die Kosten für den Flughafenneubau und der Unterhalt einer Garnison von 3000 Mann enthalten. Bezogen auf jeden der 1800 Falkland-Inseln-Bewohner, die sog. "Kelpers", macht dies eine Summe von 3,9 Millionen Mark aus. Für den militärischen Schutz der Inseln veranschlagt das britische Verteidigungsministerium die künftigen Ausgaben mit jährlich 100 Millionen Pfund oder umgerechnet 280 Millionen Mark, bezogen auf jeden der "Kelpers" also mit jährlich 155000 Mark. Im Angesicht der Kosten in solcher Höhe drängt sich die Frage auf, in welchem Interesse der Falkland-Krieg letztlich geführt wurde. Denn hätte London jedem Falkland-InselBewohner, der sich mit einem Souveränitätswechsel nicht einverstanden erklären wollte, nur die Summe von 1 Mill. Mark als Entschädigung und zur künftigen Existenzsicherung angeboten, so wäre mit großer Wahrscheinlichkeit dreierlei erreicht worden: der Krieg wäre vermieden worden, die umstrittene Grundsatzfrage des Selbstbestimmungsrechts hätte sich nicht gestellt und Ausgaben in Milliardenhöhe wären nicht entstanden. So aber wecken die verlautbarten Summen Zweifel, ob die politischen Entscheidungsinstanzen vor Beginn der Feindseligkeiten überhaupt rationale Kosten-Nutzen-Berechnungen angestellt haben und ob die gegebenen Verhandlungsmöglichkeiten von ihnen in extenso ausgeschöpft worden sind. Es sind diese Defizite der Politik, die den kritischen Zeitbeobachter skeptisch stimmen und die den Falkland-Krieg über den Rang eines zeitbedingten und regionalen Konfliktes zweier Staaten hinausheben.

Uber die Lehren, die aus dem weniger atypisch als überraschend verlaufenden FalklandInseln-Konflikt gezogen werden können, unterrichtet ein von Alberto R. Coll und Anthony $C$. Arend herausgegebener Sammelband unter dem Titel: "The Falklands War Lessons for Strategy, Diplomacy and International law". Coll als Völkerrechtler und Arend als Politik wissenschaftler haben 15 fundierte Beiträge von Fachautoren zusammengestellt, die in einer multidisziplinaren und vergleichenden Betrachtung des Konfliktverlaufs unterschiedliche Perspektiven herausgearbeitet haben. Sie sind wertvoll, um generelle Aussagen zur Steuerung politischer Konflikte zu gewinnen, vor allem, um Eskalationsgefahren nach dem Muster des Falkland-Krieges zu vermeiden.

\section{Ungenutzte Verhandlungschancen}

Schon die Diplomatiegeschichte des Falkland-Konflikts macht deutlich, welche realen Verhandlungsmöglichkeiten noch bis unmittelbar vor Ausbruch der Feindseligkeiten zwischen Argentinien und Großbritannien bestanden haben, aber ungenutzt geblieben sind. Douglas Kinney zeigt in einem Betrag auf, wie London, nachdem es mehr als 120 Jahre den argentinischen Anspruch auf die Falkland-Inseln in unregelmäßigen Zeitab- 
ständen zurückgewiesen hatte, erstmals in den Jahren 1965/68 zu einem prinzipiellen Souveränitätsverzicht bereit war, diesen Schritt aber nicht vollzog, weil es für sich selbst die Vorfrage noch nicht beantwortet hatte, in welcher Weise es die Inselbevölkerung an der Entscheidung beteiligen sollte. Zur Diskussion stand damals folgende Alternative: entweder die "Wünsche" der "Kelpers" zu "berücksichtigen" ohne ihnen selbst ein Mitspracherecht einzuräumen, oder ihnen ein Recht auf ausdrückliche Zustimmung zum Souveränitätstransfer zuzugestehen. Weder vom Völkerrecht noch vom britischen Verfassungsrecht her war eine der beiden Vorgehensweisen vorgeschrieben: Das "Selbstbestimmungsrecht der Völker", wie es die UN-Resolution 1514 festgeschrieben hat, traf in seinen tatsächlichen Voraussetzungen auf die "Kelpers" nicht zu. Auf der anderen Seite war der Status der Falkland-Inseln als britische Kronkolonie mit begrenzten Selbstverwaltungsrechten nicht so ausgestaltet, daß er in der Frage der politischen Neuordnung des Inselterritoriums London in eine bestimmte Pflichtenstellung gegenüber den "Kelpers" brachte. Argentinien forderte die baldige Souveränitätsübertragung ohne ausdrückliche Befragung der Bevölkerung, also eine status quo-Änderung, die im Kern ihrer Aussage von den britischen Vorstellungen nicht so weit entfernt war, daß sie nicht mit ihnen hätte in Einklang gebracht werden können.

Die britische Regierung zog es jedoch vor, zunächst durch praktische Schritte der Verbesserung der Post-, Nachrichten- und Verkehrsverbindung zwischen der Inselgruppe und dem Festland auf der Grundlage eines entsprechenden argentinischen Entgegenkommens die Souveränitätsfrage für die weiteren Verhandlungen zu entschärfen. Obgleich sich die britischen Hoffnungen in der Folgezeit durch mehrere bilaterale Vertragsabschlüsse zu erfüllen schienen, stand die Souveränitätsfrage auch weiterhin so im Vordergrund der Diskussion, daß sie sich durch Erfolge an der Front der praktischen Ausgestaltung der bilateralen Beziehungen nicht entschärfen ließ. Dazu hat auch der britische Vorschlag von 1974, ein "Condominium" einzurichten, beigetragen. Das Mißtrauen der "Kelpers" gegenüber den britischen Absichten wurde so stark, daß Großbritannien sich genötigt sah, den Verhandlungsgegenstand in Richtung auf eine mehr wirtschaftliche Zusammenarbeit zu verlagern. Gemeinsame britisch-argentinische Entwicklungsprojekte auf den Falkland-Inseln wollte jedoch Argentinien nicht akzeptieren, wenn damit ein Zurückweichen Großbritanniens hinter die Linie der einmal gezeigten Konzessionsbereitschaft in der Souveränitätsfrage verbunden sein sollte. Um dieses Ziel nicht aus dem Auge zu verlieren, schlug Argentinien einen Souveränitätstransfer auf sich mit einem zugleich vereinbarten und zeitlich befristeten "lease back" der Souveränität auf Großbritannien vor.

In dieser Situation, als noch das argentinische Angebot einer "Rückverpachtung" der Falkland-Inseln an Großbritannien im Raum stand, hat ein Report, den die britische Regierung durch Lord Shackleton erstellen ließ und mit dem die Perspektiven einer stärkeren wirtschaftlichen Nutzung der Inselgruppe und der angrenzenden Meeres- und Meeresbodenzonen eruiert werden sollten, auf die argentinisch-britischen Verhandlungen lähmend gewirkt. Argentinien beunruhigten die Feststellungen des Shackleton-Reports in einem Maße - zu Recht oder Unrecht, läßt Kinney unbeantwortet -, daß Ver- 
handlungen für lange Zeit unmöglich wurden. Der bewaffente Zwischenfall vom Februar 1976 war Ausdruck des angespannten Verhältnisses beider Staaten. Erst gegen Ende des Jahrzehnts, 1979/80, waren beide Parteien willens und in der Lage, die Verhandlungen in New York wieder aufzunehmen und den argentinischen "lease back"-Vorschlag zu beraten. Militärische Zwischenfälle und Provokationen auf beiden Seiten ließen diesen Vorschlag jedoch für eine Realisierung noch nicht reif erscheinen.

War es in den Jahren 1970/80 auf britischer Seite noch die überaus vorsichtige und zögerliche Behandlung und Modalitäten des Souveränitätstransfers, an der die Verhandlungslösung scheiterte, so kennzeichnete in den folgenden Jahren eine zunehmende Verhärtung der Grundpositionen auf beiden Seiten die weitere Entwicklung. Sie machte eine Verhandlungslösung schwieriger und leitete letztlich in die Phase der bewaffneten Auseinandersetzung über. Dies ist umso tragischer, als noch im Februar 1982, also kurz vor Ausbruch der Feindseligkeiten, in der Souveränitätsfrage ein Kompromiß möglich erschien: Die argentinischen Unterhändler hatten eine "offene" Tagesordnung für die weiteren Verhandlungen zugestanden, während Großbritannien seine Bereitschaft erklärt hatte, noch vor Ende des Jahres 1982 ein Abkommen anzustreben. Diesen Kompromiß stimmte jedoch die argentinische Junta Anfang März 1982 nicht zu.

Die definitive Absage Argentiniens an eine Verhandlungslösung, die nicht sofort und uneingeschränkt den Souveränitätstransfer auf Argentinien einleitete, gab den nachfolgenden Aktionen, und waren sie auch noch so unbedeutend in der Relation zu den Folgen eines bewaffneten Zusammenstoßes oder mißverständlich und unbeabsichtigt in der mit ihnen verfolgten Intention, ein Gewicht, das die konkreten Schritte in Richtung auf den bewaffneten Austrag des Streites geradezu zwangsläufig vorbereitete. Dies gilt nicht nur für die auf eine sofortige Lösung drängende argentinische Politik, sondern ebenso für das britische Ziel der kompromißlosen Wiederherstellung des Status quo ante auf der Grundlage der bilateralen Vertragsbeziehungen von 1971, verbunden mit der Forderung nach Abschluß eines Interimabkommens, das die endgültigen Ergebnisse von Verhandlungen in der Souveränitätsfrage nicht vorwegnehmen durfte. An dieser verschärften Grundsatzposition hat die britische Regierung bis heute festgehalten. Die britische Diplomatie zog zwar in jeder Phase des Konflikts Verhandlungen einer bewaffneten Auseinandersetzung vor, jedoch nicht um den Preis der Aufgabe ihrer Grundsatzforderung. London reduzierte damit seine Haltung auf ein "alles oder nichts", wie dies für jede der militärischen Konfliktaustragung unmittelbar vorausgehende Phase der Auseinandersetzungen zweier Konfliktparteien typisch ist.

\section{Der amerikanische Rückhalt}

Die argentinische und die britische Immobilität finden ihre Entsprechung in den gescheiterten Vermittlungen des peruanischen Präsidenten Belaunde-Terry und des amerikanischen Sonderbotschafters Haig. Sie werfen ein scharfes Licht auf die Verantwortung beider Streitparteien für den Ausbruch der Feindseligkeiten, aber auch auf die Mitverant- 
wortung der Vereinigten Staaten für die Nichtverhinderung des bewaffneten Konflikts. Haig gelang es nicht, die britische Regierung zu Teilkonzessionen in der Souveränitätsfrage zu bewegen. Den peruanischen Vermittlungsvorschlag torpedierte die britische Regierung gleichsam selbst durch die Versenkung des argentinischen Schlachtschiffes "Belgrano" in internationalen Gewässern.

Die kompromißlose Haltung Londons beruhte auf der Zuversicht, daß die Vereinigten Staaten Großbritannien nicht im Stich lassen würden. In der permanent wiederholten Betonung der "Legitimität" der Forderung nach kompromißloser Wiederherstellung des status quo ante auf den Falkland-Inseln kam die britische Zuversicht in den amerikanischen Beistand unmißverständlich zum Ausdruck. Die Regierung in Washington tat ihrerseits nichts, um London in diesem Glauben zu erschüttern. Die verdeckte diplomatische und später die indirekte militärische Unterstützung, die Washington seinem Verbündeten im Südatlantik zuteil werden ließ, war der Grund dafür, daß sich die Vermittlungsaktion Haigs nicht den Anschein jener Unparteilichkeit geben konnte, die für den Erfolg der Vermittlung eine unabdingbare Voraussetzung war.

Kinney betont allerdings auch, daß die britische Regierung deshalb mit so hohem Einsatz spielen konnte, weil sich Buenos Aires in der Souveränitätsfrage so unnachgiebig zeigte. Die argentinische Regierung verschaffte Großbritannien dadurch eine, wenn auch völkerrechtlich fragwürdige, Rechtfertigung, die Eskalationsleiter zu besteigen - zu sehen in der Einrichtung einer 200 Seemeilen tiefen Sperrzone um die Falkland-Inseln für Schiffe aller Nationen, einschließlich einer entsprechend ausgedehnten Sperrzone für Luftfahrzeuge. Darüber hinaus verschaffte Buenos Aires London die in diesem Zusammenhang wichtige Legitimation, alle Antennen bewußt eingezogen zu halten, um nicht mit Vorschlägen konfrontiert zu werden, welche London hätten veranlassen können, von bestimmten militärischen Eskalationsschritten Abstand zu nehmen. So hat die britische Regierung noch unmittelbar vor Versenkung des argentinischen Schlachtschiffes "Belgrano" die peruanische Initiative nicht wirklich zur Kenntnis genommen, als sie sie guthieß, aber zugleich mit der unannehmbaren Forderung der uneingeschänkten Wiederherstellung der britischen Besitzverhältnisse auf den Falkland-Inseln verband und damit ablehnte.

\section{Die UNO als "Legitimationsstifter"}

Die gescheiterten Vermittlungen der USA und Perus sind für Inis L. Claude Anlaß, einen bemerkenswerten Gedanken in die Diskussion einzubringen. In seinem Beitrag über die Bemühungen der Vereinten Nationen, die Streitparteien vor der Eskalation zu bewahren, unterscheidet er zwischen dem Konflikt in der Phase der friedlichen Streitbeilegung und dem Konflikt nach Ausbruch der Feindseligkeiten. Während in der ersten Phase die Neutralität der UNO geboten erschien, galt dies nicht in der zweiten Phase. Der Úbergang von der friedlichen Streitbeilegung zur Gewaltanwendung im Falkland-Konflikt habe für die UNO eine Eingreifverpflichtung im Rahmen der Vorschriften der Charta 
über die kollektive Sicherheit begründet, jedoch nicht in dem Sinne, daß es zu UN-autorisierten Zwangsmaßnahmen hätte kommen müssen. Vielmehr konnte dies auch in der Form geschehen, daß der Sicherheitsrat als "Legitimationsstifter" ("disperser of collective legitimacy“) für Zwangsmaßnahmen im Rahmen des in Art. 51 festgeschriebenen Rechts der individuellen oder kollektiven Selbstverteidigung auftrat.

Nach Inis Claude stand dabei der Sicherheitsrat vor der Frage, für welchen Frieden er Legitimation erteilen wollte. Wenn "Frieden" im Sinne der Vorschriften Art. 39 ff. den status quo zur Voraussetzung hat, dann konnte darunter sowohl der Argentinien begünstigende status quo nach der argentinischen Landung als auch der Großbritannien begünstigende status quo vor der argentinischen Landung verstanden werden. Angesichts der unentschiedenen Grundsatzfrage wurde die UN-Vermittlung von beiden Parteien instrumentalisiert: die UNO wurde nicht für das Ziel der Streitbeilegung, sondern für das des Sieges über den Gegner eingeschaltet, um der eigenen Sache, von beiden Seiten aus dem Recht auf Selbstverteidigung glorifiziert, den Segen der Staatengemeinschaft zu verschaffen. In diesem Ringen um Legitimität hat Großbritannien, nicht Argentinien, den Sieg davon getragen. Der Irrtum Argentiniens bestand darin, nicht erkannt zu haben, daß die UNO den Streitfall nicht als einen unter der UN-Resolution 1514 zu entscheidenden Dekolonisierungsfall einschätzte, sondern als einen Souveränitätskonflikt zweier Staaten, der nur durch Verhandlungen zu lösen sei.

Dementsprechend ist Argentinien vom Sicherheitsrat als "Aggressor" verurteilt worden. Die darin eingeschlossene Legitimität der britischen Zwangsmaßnahmen als Ausübung des Rechts der Selbstverteidigung wird von Claude aber nicht dahin ausgelegt, als wollte der Sicherheitsrat damit alle britischen Aktionen zur Wiederherstellung des status quo ante gutheißen. Nur der Wortlaut der Sicherheitsrats-Res. 502, der sich unmittelbar auf die argentinische Invasion im Zeitpunkt ihres Ablaufes bezog, vermochte den Abstimmungserfolg im Sicherheitsrat zu sichern. Er setzte voraus, daß Argentinien der Aufforderung aus der Resolution 502 Folge leistet. Soweit Argentinien diese Resolution aber nicht befolgte und Großbritannien mit Zwangsmaßnahmen gegen das argentinische fait accompli auf den Falkland-Inseln zuwartete, verlor die Resolution 502 ihr legitimierenden Wirkungen für eine Zwangsdurchsetzung des britischen Begehens auf Wiederherstellung des status quo ante. Damit blieb Großbritannien kein anderer Weg als in erneuten Verhandlungen mit Buenos Aires - mit oder ohne Einschaltung der UNO - nach einer Lösung zu suchen, ohne noch die Möglichkeit zu haben, die Resolution auch für ein bewaffnetes Vorgehen gegen Argentinien zu verwenden.

Die in der UNO zunehmend lauter gewordene Forderung nach Einstellung aller militärischen Handlungen wirkte sich unter dem britischen Zuwarten immer mehr zu einer gegen Großbritannien gerichteten Forderung aus, den von Argentinien geschaffenen Verletzungstatbestand als neuen status quo zu akzeptieren und die verbliebenen of fenen Fragen in Verhandlungen zu lösen. Die Sicherheitsrats-Resolution 505 ist weitgehend Ausdruck dieser veränderten Einstellung der UN-Mitglieder. Der Stimmungswandel in der UNO kündigte sich in der EG an (Ausscheren Irlands und Italiens aus der Front der Argentinien wirtschaftlich boykottierenden EG-Staaten) und setzte sich im Sicherheitsrat 
fort, wo die britischen Änderungswünsche zum panamesischen Resolutionsentwurf (2. 6. 1982) nicht mehr berücksichtigt waren, so daß dieser in der Schlußabstimmung nur noch durch das britische Veto zu Fall gebracht werden konnte.

Das Ergebnis, daß der "Selbstverteidiger" je eher als "Aggressor" erscheint, je länger er mit einer bewaffneten Reaktion zuwartet, führt Claude auf die Haltung der Vereinten Nationen zurück, die die Legitimationswirkungen ihrer Entscheidungen möglichst zu begrenzen trachten, sobald einseitige Zwangsmaßnahmen in Rede stehen. Der Sicherheitsrat wendet Art. 39 nicht wort- oder sinngemäß an und segnet deshalb Maßnahmen der individuellen Selbstverteidigung als Ersatz für nicht stattgefundene kollektive Sanktionen nur in engen Grenzen ab - eine Haltung die Claude in dem Satz zusammenfaßt, die UNO will den "Frieden", aber nicht um den Preis seiner Verletzung.

\section{Die OAS zwischen UN-Verurteilung und EG-Sanktionen}

Eine große Diskrepanz zwischen Anspruch und Wirklichkeit kennzeichnet die OAS, sobald sie sich aufgefordert sieht, zu Gewaltmaßnahmen innerhalb der lateinamerikanischen Hemisphere Stellung zu nehmen. Die Gründe für dieses Phänomen liegen weniger in der verbreiteten Skepsis vieler OAS-Mitglieder gegenüber kollektiven Zwangsmaßnahmen als in der historischen Tatsache, daß die lateinamerikanischen Staaten selten zu jener Solidarität gefunden haben, in der sie eines ihrer Ideale sehen. Der übereinstimmende Wunsch, europäischen Interventionsversuchen gemeinschaftlich zu begegnen, stand schon immer in einem unauflösbaren Gegensatz zu dem anderen Integrationsziel lateinamerikanischer Staaten, inneramerikanischen Streitigkeiten, vor allem im Bereich der territorialen Grenzziehung, durch eigene regionale Organe zu entscheiden. Seit den Tagen der "Heiligen Allianz" und den gegen diese ergriffenen Vertrags- und Organisationsinitiativen Simon Bolivars ist dieser Widerspruch nicht wirklich aufgelöst worden. Häufig haben auch die Vereinigten Staaten, das einflußreichste OAS-Mitglied, den amerikanischen Integrationsanstrengungen im Wege gestanden. Ihre lange Tradition als Interventionsmacht in Lateinamerika ist einer der Gründe. Ihre Politik, die OAS immer dann zu mobilisieren, wenn es "kommunistische" Bedrohungen vom Kontinent fernzuhalten gilt, ein anderer. Selten haben die USA aber die OAS für die Entschärfung lateinamerikanischer Spannungen eingesetzt.

Vor diesem historischen Hintergrund hatte es die OAS, wie Srilal Perera aufzeigt, im Falkland-Konflikt schwer, eigenes Profil zu gewinnen. Noch schwieriger wurde ihr diese Aufgabe, als sie sich zwischen UN-Verurteilung und EG-Sanktionen eingeklemmt sah. Das Ziel der OAS, die Anerkennung des argentinischen Souveränitätsanspruchs durchzusetzen, scheiterte an zwei Faktoren: zum einen an dem Wunsch einiger OAS-Mitglieder, darunter solcher, die als frühere britische Kolonien mit Großbritannien sympathisierten, Argentinien als "Aggressor" verurteilt zu wissen, zum anderen an der Haltung der Vereinigten Staaten, die sich nach dem Scheitern der Haig-Mission offen auf die Seite Großbritanniens stellten. Die USA hatten ihre zunächst nach außen gezeigte Neutra- 
lität aufgegeben, obgleich weder die Sicherheitsrats-Resolution 502 noch der amerikanische Sicherheitspakt von 1947 (Rio-Pakt) sie dazu verpflichtete. Anstatt ihre Doppelstellung als OAS-Mitglied und Supermacht mit universalem Verantwortungsanspruch für eine glaubwürdige Vermittlung im Falkland-Konflikt zu verwenden, gaben sie sich im Wege einer "Úberinterpretation" der Resolution 502 selbst eine Handlungsanweisung, um Großbritannien bei dessen Anstrengungen, die Falkland-Inseln wieder zu erobern, zu unterstützen (kein Fall einer vonaußerhalb des Kontinents unternommenen Aggression!).

\section{Der Einfluß der internationalen Rechtsprinzipien auf den Falkland-Konflikt}

Konnte der Falkland-Konflikt weder durch die Konfliktparteien selbst noch durch die zwischenstaatlichen Konfliktregelungsmechanismen (UNO, OAS und Vermittlung dritter Staaten) politisch entschärft werden, so bleibt die Frage, welchen Einfluß die internationalen Rechtsprinzipien auf den Verlauf des Konflikts gehabt haben.

Thomas M. Franck mißt den Rechtsprinzipien eine dominierende Rolle im politischstrategischen Kalkül der Staaten bei. Er meint aber, daß sie in der politischen Praxis nicht immer beachtet worden seien. Als Beispiele nennt er den Goa-Konflikt (1961), den Streit um Papua-Neuguinea (1963) sowie den Ost-Timor- und den West-Sahara-Konflikt (1975). Die Behandlung, die diese Konflikte seitens der Dritten Welt erfuhren, habe die argentinische Junta zu der Annahme verführt, im Fall eines bewaffneten Vorgehens diese Staatengruppe in ihrer großen Mehrheit hinter sich zu haben.

Mit der argentinischen Fehleinschätzung der Reaktion der Dritten Welt war etwas verloren gegangen, das den internationalen Rechtsprinzipien im Regelf all auch beigemessen wird: die Fähigkeit, potentielle Rechtsbrecher vor rechtsbrecherischen Handlungen abzuschrecken. Nach der Landung argentinischer Streitkräfte auf den Falkland-Inseln konnte speziell das Agggressionsverbot diese Wirkung gegenüber Großbritannien nicht mehr entfalten. Dafür gewann Großbritannien eine Legitimation, die Glaubwürdigkeit dieses für das friedliche Zusammenleben der Staaten grundlegenden Rechtsprinzips wiederherzustellen. Wenn auch im Falkland-Krieg einiges dafür spricht, daß weniger für dieses Prinzip als für außerrechtliche Ziele und Interessen gefochten wurde, so ist doch die Wiedereroberung der Falkland-Inseln von Großbritannien, aber auch von den USA gerade aus Rechtsgründen gerechtfertigt worden. Nur unter diesem Aspekt konnten die von Großbritannien aufgewendeten Milliardensummen trotz eines absurden KostenNutzen-Verhältnisses überhaupt einer breiteren Offentlichkeit zugemutet werden.

Ausgehend von der Erfahrung, daß Recht und Unrecht sich selten eindeutig der einen oder anderen Partei zuordnen lassen, stellt Alberto R. Coll die seit dem Ende des Ersten Weltkrieges unternommenen Anstrengungen der Staaten, zwischen dem Aggressor und dem Nichtaggressor eine Trennlinie zu ziehen, als auffälligstes Moment der internationalen Rechtsentwicklung heraus. Die Uberprüfung traditioneller und heutiger Aussagen zur Frage der erlaubten und der unerlaubten Kriegsführung bestätige, daß die interna- 
tionalen Normen heute stärker als in der Vergangenheit unter dem Prinzip des Verbotes der gewaltsamen Veränderung des territorialen Status quo gesehen werden.

Für den Falkland-Konflikt folgert Coll daraus, daß Großbritannien, trotz illegalen Erwerbsaktes, wegen seines langandauernden Besitzes der Falkland-Inseln einen Anspruch darauf hatte, daß Argentinien seine Forderungen nicht im Wege der Gewalt durchsetzte. Nur die Verhandlungslösung war die "funktionale" und "legitime" zugleich. Der britische Anspruch fand seine Bestätigung in zahlreichen "Post World War II political and territorial arrangements", in denen die fehlende de iure-Anerkennung durch eine Formalisierung von de facto-Situationen ersetzt worden ist. Allerdings meint Coll, daß die Staatenpraxis noch nicht überzeugend beweise, daß das Aggressionsverbot ebenso autoritativ angewandt werde wie andere Normen des internationalen Rechts. Daraus folgert er, daß die Wiederherstellung des normativen Gehalts des Aggressionsverbotes eine realistische Anpassung des UN-Standards "nach unten" notwendig machen könnte. Ob eine Abschwächung der normativen Bindungswirkung oder der Tatbestandsvoraussetzungen des Aggressionsverbotes die zwischenstaatliche Gewaltenanwendung reduziere, bleibt jedoch solange fragwürdig, als sie nicht durch eine Verstärkung der institutionellen Konfliktregelungsmechanismen ausgeglichen wird. Gerade das britische Veto im Sicherheitsrat gegen den panamesischen Resolutionsentwurf vom Juni 1982 beweist, wie wenig den Staaten daran gelegen ist, die autonome Konfliktregelung unter Verzicht auf selbst definierte Interessenverfolgung anzunehmen.

Anthony Arend beschäftigt die Frage, welche Legitimität den Charta-Prinzipien unter den heutigen politischen Bedingungen fundamentaler Rechtsverstöße noch zukomme. Ausgehend von der These, daß die Methoden des Konfliktmanagements sowohl in der Form der kollektiven Zwangsmaßnahme (Art. 39) als auch in der modifizierten Form der "Peacekeeping operation" (mit Zustimmung der betroffenen Staaten) versagt haben und auch der Mechanismus des friedlichen Wandels der Legitimität entbehre, stellt Arend die Frage, ob sich nicht neue Normen an deren Stelle entwickelt haben. Eine solche Normneubildung erkennt er in der Verpflichtung, die anzustrebende Streitbeilegung nicht im Ausbruch von Feindseligkeiten enden zu lassen, sondern sie über diese Grenzlinie zwischen Frieden und Krieg hinaus fortzusetzen, bis eine Streitbeendigung erreicht ist. In der Resolution 502, die beide Seiten dazu auffordere, "eine diplomatische Lösung zu suchen" und ebenso in den vermittelnden Aktivitäten Alexander Haigs, BelaundeTerrys und des UN-Generalsekretärs Javies Perez de Cuellar sieht Arend Anzeichen einer solchen Normneubildung.

Die Frage, die Arend aufwirft, ist, vom Friedensziel der Vereinten Nationen aus gesehen, stimmig, jedoch nicht ausreichend beantwortet. Es bleibt die Frage nach dem Grad der politischen Umsetzung einer solchen Norm in der politischen Praxis. Sie würde eine stärkere Identifikation des staatlichen Akteurs mit den Zielen der Vereinten Nationen voraussetzen, als es noch der Fall ist. Es ist nicht ausgemacht, ob die Haig-Mission den politischen Kompromiß zwischen Argentinien und Großbritannien nicht in erster Linie aus der Sorge um die Erhaltung des westlichen Verteidigungsbündnisses angestrebt und so gesehen nur den Ausgleich zwischen einer intakten NATO-Partnerschaft und der 
Monroe-Doktrin gesucht hat. An einer Stärkung der UNO zulasten des Selbstverteidigungsrechts waren die USA damals weniger denn je zuvor interessiert. Immerhin räumt Arend ein, daß ein Zurückgehen auf die Normenstruktur der Vor-Völkerbundzeit, als die Selbsthilfe noch weitgehend unangefochten war, die schlechteste aller Lösungen wäre, um die Staatenwelt aus dem Gewaltdilemma herauszuführen.

\section{Pragmatische Lösungen für den Souveränitätskonflikt}

Die Hoffnungen Argentiniens, im Falkland-Konflikt mit Großbritannien unter den Staaten eine überzeugende Sympathiemehrheit zu finden, haben sich nicht erfüllt. Die Spekulation, eine Verbindung seines Zieles einer status quo-Änderung mit dem Gedanken der Dekolonisierung könne eine Selbsthilfe-Aktion nach klassischem Vorbild rechtfertigen, erfüllte sich nicht. Andererseits ist das argentinische Vorgehen auch ein Symptom für das verbreitete Mißtrauen vieler Staaten in die Fähigkeit der Vereinten Nationen, einen friedlichen Wandel in einer Reihe von Territorialkonflikten einzuleiten.

Angesichts der Stagnation, in die viele Verhandlungen um eine Änderung des status quo geraten sind, werden pragmatische Lösungen immer dringender. Im Falkland-Konflikt ergibt sich die Notwendigkeit einer pragmatischen Lösung vor Klärung des Souveränitätskonfliktes auch aus dem Umstand, daß Großbritannien zuletzt die Seegrenzen der Inselgruppe auf 150 Seemeilen festgesetzt hat und daß sich diese Grenzziehung in Teilen mit der älteren 200-Seemeilen-Zone Argentiniens zum Schutz seiner Küstenfischerei überschneidet.

David A. Colson sieht in den Regelungen, die interessierte und betroffene Staaten in Spitzbergen (1925) und in der Antarktis (1959) sowie in der Meerenge von Torres (1978) vorgenommen haben, Ansatzpunkte für Konfliktlösungen auch im Falkland-Konflikt. Diese Regelungen beruhen alle auf dem Modell der Ausklammerung beziehungsweise der Entschärfung der Souveränitätsfrage auf der Basis interessengerecht ausgehandelter Kompromisse.

In Spitzbergen wurde eine pragmatische Lösung gefunden, weil Norwegen die wirtschaftlichen Interessen an der Ausbeutung der Rohstoffvorkommen in rechtlich bindender Form anerkannte und dieses Anerkenntnis in seiner innerstaatlichen Gesetzgebung berücksichtigte. Norwegen stimmte ferner einer vollständigen Entmilitarisierung dieser Inselgruppe zu. Darauf erhielt es die formelle Souveränität über Spitzbergen übertragen, die von Stund an nicht mehr im Streit stand.

In der Antarktis wurde der Kompromiß unter Ausklammerung der wechselseitigen Souveränitätsansprüche, die aufrechterhalten bleiben, in einer prinzipiellen freien Nutzung des Eisgebietes für wissenschaftliche Zwecke seitens der Antarktis-Vertragsstaaten gefunden. Auch hier hat die Entmilitarisierung des Vertragsgebietes das Abkommen erleichtert. Im dritten Beispielfall war es der Kompromiß zwischen Papua-Neuguinea und Australien in der Frage der ehtnischen und lokalen Bedürfnisse und Besonderheiten der Fischfang treibenden Inselvölker der Torresstraße, der die bis dahin strittige Bestim- 
mung der Seegrenzen ermöglichte. Dabei wurde die Jurisdiktion nicht so festgeschrieben, daß sie nicht auch dort noch Raum für funktionale Problemlösungen läßt, wo das Abkommen die Parteien im Stich läßt. Colson plädiert für einen "modus vivendi" im Falkland-Konflikt, der allerdings so ausgestaltet sein müsse, daß bestimmte Ereignisse oder Vorkommnisse nicht die Ansprüche der einen oder anderen Partei präjudizieren.

\section{Status quo und historische Rechtstitel}

Die argentinische Politik der letzten zwei Jahrzehnte im Falkland-Streit zielte nicht primär auf eine Feststellung der rechtlichen Eigentumsverhältnisse, sondern auf eine - allerdings rechtlich bindende - Änderung des status quo. Trotzdem hat Argentinien in diesem Konflikt auch auf historische Rechtstitel zurückgegriffen und seinen Anspruch auf status-quo-Änderung mit diesen Titeln zu begründen versucht, vielleicht auch deshalb, weil das bewaffnete Vorgehen aus einer rationalen politischen und ökonomischen Sicht der Dinge so wenig überzeugend erschien.

Argentinien leitet seinen Anspruch auf Souveränitätstransfer aus spanischen Rechtstiteln des 18. Jahrhunderts her sowie aus einer behaupteten Illegalität des britischen Erwerbsaktes vom Jahre 1833 im Zusammenhang mit den Auflösungserscheinungen des spanischen Kolonialreiches zu Beginn des 19. Jahrhunderts. Demgegenüber verweist London auf die britische Entdeckungsgeschichte, die bis ins 16. Jahrhundert zurückreicht, sowie auf die Tatsache seines annähernd 150 Jahre währenden, wenn auch von Argentinien bestrittenen Besitzes. Mit den historisch-rechtlichen Argumentationen, so wenig sie unter den Kategorien des heute geltenden Völkerrechts eine status quo-Änderung begründen können, setzt sich Alfred P. Rubin auseinander. Seine Argumentation kann jedoch verständlicherweise nicht mehr als eine summarische Zusammenfassung und Bewertung des höchst komplizierten Geschehens um die Inbesitznahme der Falkland-Inseln und ihrer häufigen Besitzwechsel sein.

Rubin sieht in der Frage, ob Großbritannien sich mit den Falkland-Inseln 1833 eine "res nullius" angeeignet habe, als es die zuvor von den Spaniern verlassene und von einem amerikanischen Kriegsschiff anläßlich eines Streites um Fangrechte in den Falkland-Insel-Gewässern geplünderte Inselkolonie wiederbesetzte, nicht den entscheidenden Hinweis, der britische Rechte begründen könnte. Diese Rechte seien auch nicht durch die Schiedssprüche im Insel-Palmas-Streitfall (1928) und im Insel Clipperton-Streitfall (1931) bestätigt worden. Auch die Entscheidung des Internationalen Gerichtshofes im Streit um die Ärmelkanalinseln (1953) gebe für den Streit um die Eigentumsrechte an den Falkland-Inseln nichts her. Die internationale Spruchpraxis biete keine Anhaltspunkte, den tatsächlichen Besitz für das maßgebende Kriterium zur Entscheidung der Rechtsfrage zu halten, wenn der Besitz auf einen Erwerbsakt beruhe, gegen den protestiert wurde. Der britische Standpunkt werde noch durch eine Tendenz in der internationalen Spruchpraxis geschwächt, die den vermutlich "besseren" Anspruch als lückenlos nachgewiesen ansieht und bestätigt und dem Gegenanspruch, wenn er auch vernünftig 
erscheint, eine vollständige Abfuhr erteilt. Daraus folgert Rubin, daß Argentinien vor einer internationalen Spruchkammer nicht sicher sein könnte, wie sein Titel, den es durch Eroberung verloren hat und an dem es nur durch eine unterschwellige Drohung der Gewaltanwendung zur Abwehr einer "Gegen-Eroberung" festhält, bewertet würde.

Wegen des unsicheren Ausgangs eines internationalen Spruchverfahrens waren beide Parteien auch nicht bereit, den Falkland-Konflikt in einem objektivierten Verfahren einer Prüfung zu unterziehen. Die UN-Charta verpflichtet die Parteien eines Streites nicht dazu, eine gerichtliche oder schiedsrichterliche Lösung zu suchen. Vielmehr verpflichtet die Charta die Parteien nur darauf, vinternationale Streitfälle mit friedlichen Mitteln so zu regeln, daß der Weltfriede, die internationale Sicherheit und die Gerechtigkeit nicht gefährdet werden" (Art. 2 Ziff. 3 der UN-Charta). Es war deshalb in erster Linie der Verstoß Argentiniens gegen das Verhandlungsgebot und weniger der Verstoß gegen das Gewaltverbot (Art. 2 Ziff. 4), der die USA und andere Staaten dazu brachte, Großbritannien in seinem Wunsch, seine Besitzansprüche wiederhergestellt zu sehen, zu unterstützen.

Ausführlicher, als es Rubin verständlicherweise in seinem Beitrag vermag, behandelt Rudolf Dolzer die historischen Rechtsfragen des Falkland-Konflikts. In seiner 1986 unter dem Titel "Der völkerrechtliche Status der Falkland-Inseln (Malvinas) im Wandel der Zeit" erschienenen Monographie trägt er alles zusammen, was die Parteien hierzu vorgebracht und was die bisherigen Untersuchungen dazu ergeben haben. ${ }^{1}$ Im Ergebnis decken sich Dolzers Ausführungen mit denen Rubins, im einzelnen gelangt Dolzer jedoch nicht zu neuen Aussagen. Dies gilt auch für seinen Versuch, den britischen Erwerbsakt von 1833 vor dem Hintergrund des spanischen Schweigens in den Jahren nach 1820 in seinen Rechtswirkungen zu relativieren.

Dolzers Ansatz, der den La Plata-Staat bereits in seiner Anfangsphase als ein vollendetes Staatswesen und daraus folgend als unbestrittenes Völkerrechtssubjekt annimmt, erscheint wenig überzeugend angesichts einer politischen Realität, deren Veränderung zum fraglichen Zeitpunkt nicht abgeschlossen war und die in ihrer Gesamtheit, sowohl innenpolitisch als auch außenpolitisch gesehen, alles andere als gesichert galt. Dolzers Hauptirrtum besteht in der Nichtunterscheidung zwischen Legalität und Legitimität völkerrechtlich relevanten Handelns. In der Frage, wer die Verfügungsrechte an den FalklandInseln nach der Sezession der La Plata-Provinzen von Spanien besaß, die Regierung in Buenos Aires oder die Regierung in Madrid, war nicht so sehr das Schweigen Madrids als die Tatsache relevant, daß nach Völkerrecht nur Spanien als diplomatisch voll aner-

1 Vgl. hierzu auch die Monographien: H. Weber, "Falkland Islands" oder "Malvinas"? Der Status der Falklandinseln im Streit zwischen Großbritannien und Argentinien. Eine völkerrechtliche Fallstudie, Frankfurt am Main (Alfred Metzner Verlag GmbH) 1977; B. Hillekamps, Der Streit um die Falkland-Inseln, Jur. Diss. Köln 1978. Unter den zahlreichen Zeitschriftenaufsätzen sei auf folgende Beiträge hingewiesen: A.F.J. Hope, Sovereignty and Decolonization of the Malvinas (Falkland) Islands, in: Boston College International and Comparative Law Review, vol. 6, No. 2, 1983, S. 391-446; D. E. Acevedo, The U.S. Measures against Argentina resulting from the Malvinas Conflict, in: American Journal of International Law, vol. 78, 1984, S. 323-344; C.J. Moneta, The Malvinas Conflict: Some Elements for an Analysis of the Argentinian Military Regiments Decision-making Process (1976+1983), in: VRU, 1984, No. 4, S. 457-473. 
kanntes Staatswesen in den Angelegenheiten seiner Uberseeprovinzen Verfügungs- und Vertretungsmacht hatte. Demgegenüber war das Regime in Buenos Aires aufgrund der tatsächlichen Gegebenheiten, insbesondere im Hinblick auf seine innere Organisation und seine Fähigkeit, sich gegenüber den Anforderungen dritter Staaten durchzusetzen, nur als de facto-Regierung anerkannt. Zwar gab es in der politischen Absetzbewegung der lateinamerikanischen Úberseeprovinzen vom spanischen Mutterland unterschiedliche Indizien für eine neue Legitimität, die mit der spanischen nicht in Úbereinstimmung gebracht werden konnte, sie war aber im Falle des La Plata-Staates lange nach der Unabhängigkeitsausrufung noch nicht so überzeugend nachgewiesen, daß sie auch in einer entsprechenden Zahl diplomatischer Anerkennungserklärungen einen Wiederhall gefunden hätte. Im Gegenteil: der La Plata-Staat wurde ganz überwiegend als nicht voll handlungsfähig im Sinne des Völkerrechts angesehen und wurde von dritten Staaten entsprechend behandelt.

Einzig die Regierung in Washington hatte bereits eine volle diplomatische Anerkennung des La Plata-Staates ausgesprochen, doch kam es auf diesen Akt aufgrund der Außenseiterstellung der Vereinigten Staaten zu Anfang des Jahrhunderts nicht entscheidend an. Maßgebend war, welche Stellung die damaligen Großmächte, allen voran Großbritannien als eine der führenden Seemächte, gegenüber dem La Plata-Staat bezogen hatten. London aber hatte mit Buenos Aires keine vollen diplomatischen Beziehungen aufgenommen und zum Schutz seiner Handelsinteressen lediglich einen Freundschafts-, Handels- und Schiffahrtsvertrag (1825) abgeschlossen. London achtete streng darauf, keine Vertragsbeziehungen zu unterhalten, aus denen sich Rechtsfolgen ergeben könnten, die von den politischen Realitäten nicht gestützt waren. Auch Frankreich, Preußen und alle anderen europäischen Staaten von einigem Gewicht hielten sich mit Anerkennungsakten zurück.

Noch heute entspricht die Staatenpraxis, rechtliche Anerkennungswirkungen durch den Abschluß sogenannter "unpolitischer" Verträge, zu denen die Handelsabkommen und andere technische "Regierungsabkommen" gezählt werden, zu beschränken, dem geltenden Völkerrecht. Es war also nicht der Mangel einer formellen Billigung des Sezessionsvorgangs seitens der spanischen Regierung, der den Gebietserwerb an den Falkland-Inseln auf der Seite des La Plata-Staates gehindert hat - dies war schon deshalb nicht möglich, weil nach Völkerrecht die Mutterregierung dem Rebellenregime keine völkerrechtlichen Erklärungen schuldet -, sondern es war der Mangel an Effektivität auf der Seite der Sezessionsregierung in Buenos Aires, die die Inbesitznahme der Falkland-Inseln nach damaligem wie nach heutigem Völkerrecht fragwürdig erscheinen läßt. Dieser Mangel wäre durch eine ausdrückliche Zustimmung der zuständigen Stellen in Madrid oder durch eine unmißverständliche Haltung dritter Staaten (z. B. durch eine uneingeschränkte diplomatische Anerkennung des La Plata-Staates) geheilt worden. Beides war zum fraglichen Zeitpunkt nicht der Fall.

Großbritannien konnte deshalb den nicht abgeschlossenen Prozeß der Staatswerdung der spanischen Úberseeprovinzen am Rio de la Plata für sich ausnutzen und den Rechtsschein der spanischen Verfügungsrechte durch eine eigene Besitzergreifung von den Falk- 
land-Inseln zu seinen Gunsten verändern. Dabei half Großbritannien die Zeit. Auf die fehlende Zustimmung des La Plata-Staates vor dessen völkerrechtlicher Aufwertung im Friedensvertrag mit Spanien respektive vor dessen voller diplomatischer Anerkennung seitens dritter Staaten kam es nicht an, danach aber wäre die Zustimmung des Verfügungsberechtigten verspätet gewesen. Denn inzwischen war der britische Erwerbsakt seinerseits in Effektivität erwachsen und hatte ein für die Rechtsgültigkeit des britischen status quo konstitutives Element geschaffen.

Nur eine, allerdings von der Staatenpraxis bisher nicht bestätigte, Retrospektive könnte zum gegenteiligen Ergebnis führen und Dolzers Beweisführung legitimieren, d. h. den britischen Erwerbsakt trotz einem über 150 Jahre aufrechterhaltenen und unveränderten Faktum, der britischen Herrschaftsausübung auf den Falkland-Inseln, als einen fortwährenden Unrechtstatbestand disqualifizieren. Sie hätte die gedankliche Rückübertragung von Situationsmerkmalen, die erst in einer späteren Zeit nachweisbar sind, auf den Zeitraum von 1820/33 zur Voraussetzung: die innere und äußere Konsolidierung des La Plata-Staates und seine Aufnahme in den Kreis der Staatengemeinschaft durch in ihren Rechtswirkungen unmißverständliche Akte. 